\title{
Photochemistry and Photobiology
}

The official journal of the American Society of Photobiology

\section{Effect of LED Blue Light on Penicillium digitatum and Penicillium italicum Strains}

\begin{tabular}{|r|l|}
\hline Journal: & Photochemistry and Photobiology \\
\hline Manuscript ID: & PHP-2015-06-RA-0179.R1 \\
\hline Wiley - Manuscript type: & Research Article \\
\hline Date Submitted by the Author: & n/a \\
\hline Complete List of Authors: & $\begin{array}{l}\text { Lafuente, María; IATA-CSIC, } \\
\text { Alférez, Fernando }\end{array}$ \\
\hline Keywords: & LED blue light, P. digitaum, P. italicum, antifungal, citrus fruit \\
\hline & \\
\hline
\end{tabular}

\section{SCHOLARONE ${ }^{\text {w }}$ \\ Manuscripts}


4 María T. Lafuente*, Fernando Alférez ${ }^{1}$

5 Instituto de Agroquímica y Tecnología de Alimentos (IATA-CSIC). Consejo Superior de

6 Investigaciones Científicas. Av. Agustín Escardino, 7. 46980, Paterna-Valencia, Spain

7

8 *Corresponding autor e-mail : $\underline{\text { mtlafuente@iata.csic.es (María T. Lafuente) }}$

9

$10{ }^{1}$ Current address: Citrus Research and Education Center. Institute of Agricultural and Food

11 Sciences, University of Florida. 700 Experiment Station Rd, 3850 Lake Alfred, Fl, USA. 


\section{ABSTRACT}

14

15 Studies on the antimicrobial properties of light have considerably increased due in part to 16 the development of resistance to actual control methods. This study investigates the 17 potential of Light Emitting Diodes (LED) blue light for controlling Penicillium digitatum 18 and Penicillium italicum. These fungi are the most devastating postharvest pathogens of 19 citrus fruit and cause important losses due to contaminations and to the development of 20 resistant strains against fungicides. The effect of different periods and quantum fluxes and 21 of delaying light application on the growth and morphology of P.digitatum strains resistant 22 and sensitive to fungicides and of $P$. italicum cultured at $20{ }^{\circ} \mathrm{C}$ was examined. Results 23 showed that blue light controls the growth of all strains and that its efficacy increases with 24 the quantum flux. Spore germination was always avoided by exposing the cultures to high 25 quantum flux $\left(700 \mu \mathrm{molm}^{-2} \mathrm{~s}^{-1}\right)$ for $18 \mathrm{~h}$. Continuous light had an important impact on the 26 fungus morphology and a fungicidal effect when applied at a lower quantum flux (120 $\left.27 \mu \mathrm{molm}^{-2} \mathrm{~s}^{-1}\right)$ to a growing fungus. Sensitivity to light increased with mycelium age. Results 28 show that blue light may be a tool for $P$. digitatum and $P$. italicum infection prevention 29 during handling of citrus fruits. 


\section{INTRODUCTION}

Citrus fruits are subjected to various postharvest diseases that cause important economic losses. These losses are mostly due to pathogen contaminations in different strategic points in the packing-houses, such as drenchers, dip tanks, and cold storage rooms. Therefore, reducing inoculum of pathogens and maintaining good water quality in postharvest facilities is critical to reduce the incidence of decay. Penicillium digitatum (Pers.:Fr.) Sacc. (green mold) and P. italicum (blue mold) are the major pathogens of citrus fruits after harvesting. Green mold is most commonly responsible for postharvest decay of citrus, contributing up to $90 \%$ of total losses (1). The use of fungicides constitutes the most common method to control postharvest diseases in citrus fruits. At present, $P$. digitatum and $P$. italicum are controlled by synthetic fungicides. However, due to the growing concern about human health and the environment, the use of chemicals is becoming increasingly restricted and there is a trend to develop alternative methods to control postharvest diseases (2-6). In the case of citrus fruits, the search of new strategies is also relevant because of the increase of fungicide resistant strains (1) and also because of low residue tolerance, disposal of used fungicide solutions in packinghouses, chemical costs and development of organic marketing programs (7). Induction of natural resistance in the fruit by using physical and chemical treatments $(8-12)$ and biocontrol agents $(3,13)$ is one of these alternatives. Nevertheless, the efficacy of these treatments is variable and is lower than that of synthetic fungicides. Therefore, appropriate infection prevention and measures for the reduction of pathogens contamination during postharvest handling and storage of citrus fruits is critical to control disease and reduce the use of fungicides.

The antimicrobial properties of light is a research area of increasing interest, not least due to the emergence of resistance to a number of other control methods including the use 
55 of pesticides, disinfectants and antibiotics (14-18). Different reports have shown the 56 potential of UV-treatments for reducing postharvest decay caused by different fungi $57(2,5,10,19,20)$. However, UV light has limitations because of its detrimental effects to 58 workers, such as injury to the skin or eye on direct exposure. Lighting based on Light 59 Emitting Diodes (LEDs) is one of the main emerging technologies in agriculture. In 60 contrast with other illumination systems, LEDs possess features making them unique for 61 industrial applications: higher irradiation with less heat dissipation, making easier control 62 of humidity and temperature in closed environments such as storage rooms, easy 63 installation and lower energy costs. Besides, a narrower bandwidth in the light spectrum 64 achieved by this technology allows better control of the amount of light beam actually 65 exciting photoreceptors in cells (21). Studies on the potential of LED blue light on different 66 processes of agronomic interest has considerably increased during the last decade (21). 67 Moreover, it has been shown that LED blue light may control food relevant fungi (22) and other pathogens that are harmful for consumers, such as Listeria monocygenes (23), that 69 has caused important problems derived from fresh fruit contaminations during current 70 postharvest practices (24). Such contaminations can occur in different strategic points in the 71 packing-houses, such as drenchers, dip tanks or hydrocooling systems. Therefore, this 72 technology might constitute an appropriate strategy to reduce not only postharvest decay 73 caused by fungi but also the development of pathogens that can be harmful for consumers 74 during postharvest handling of horticultural crops. Nevertheless, the potential of LED blue 75 light to avoid losses of perishable crops during postharvest handling is almost unknown. In 76 fact, to our knowledge, only two postharvest studies have been performed until now that 77 suggest the industrial relevance of LED blue light reducing postharvest citrus fruit losses. 78 On one hand, it has been shown that exposure of citrus fruit to this light emerging 
79 technology, at a quantum flux of $40 \mu \mathrm{molm}^{-2} \mathrm{~s}^{-1}$ reduces infection by P.digitatum $(25,26)$

80 although this quantum flux did not induce any change in mycelium growth and sporulation

81 of the fungus in vitro. This finding indicated that blue light is able to induce resistance

82 against P.digitatum in citrus fruit. On the other hand, Liao et al. (2013) showed that a 83 quantum flux treatment of $120 \mu \mathrm{molm}^{-2} \mathrm{~s}^{-1}$ reduced in vitro fungal development. This result

84 suggests, therefore, the potential of blue light for controlling mycelial growth and/or 85 inhibiting conidia production of $P$. digitatum and that the efficacy of this physical treatment may increase with the quantum flux. Considering these results, that sanitation of packingand store-houses is essential to reduce postharvest decay (11) and the high abundance of $P$. digitatum strains resistant to the two fungicides (thiabendazole (TBZ) and imazalil) most commonly used to control decay in citrus fruit (1), the aim of this study has been to investigate the potential of LED blue light for controlling sensitive and resistant strains of $P$. digitatum and by $P$. italicum during postharvest handling of citrus fruits. To that end, we have evaluated the effect of increasing blue light quantum flux and also time of exposure to such radiations on in vitro $P$. digitatum and $P$. italicum development and morphology. Conidial suspensions of three strains from P.digitatum, two of them resistant to fungicides, and one from $P$. italicum cultured on potato-dextrose agar (PDA) were used. Furthermore, as the effectiveness of chemical and physical treatments on controlling postharvest disease may vary if the treatment is applied after spore germination (27), the efficacy of the most effective blue light treatments was evaluated at different periods after inoculating the PDA plates with the conidial suspensions.

\section{MATERIALS AND METHODS}


102 Collection of $\boldsymbol{P}$. digitatum and $\boldsymbol{P}$. italicum isolates: P. digitatum and P. italicum strains

103 used in this study were provided by Dr. González-Candelas and isolated from citrus fruit

104 with typical green or blue mold collected from different orchards or packing-houses. Most

105 strains are deposited in the Spanish Type Culture Collection (CECT). Petri plates

106 containing $20 \mathrm{ml}$ sterile PDA $\left(39 \mathrm{~g}^{-1}\right)$ were inoculated with P. digitatum (Pers.:Fr.) Sacc.

107 isolates PHI-26 (CECT 20796), which is sensitive to TBZ and imazalil, and Pd1(CECT

108 20795) and PHI-41, resistant to both fungicides, and with P. italicum isolate PHI-I (CECT

109 20909) and incubated at $20{ }^{\circ} \mathrm{C}$ for at least 7 days (8). Conidia from each isolate were

110 rubbed from the agar surface by scrapping them with a sterile spatula and transferred to 10

$111 \mathrm{ml}$ of sterile water. The resulting conidial suspensions were filtered and the concentration

112 titrated with a hemacytometer and adjusted to the desired final concentration (8).

114 Infection and evaluation of fungal growth: Petri plates of $9 \mathrm{~cm}$ diameter were inoculated

115 in triplicate for each light treatment and pathogen strain with $5 \mu 1$ of $10^{6}$ conidia $\mathrm{ml}^{-1}$. To

116 determine the effectiveness of the LED blue light treatments reducing fungal growth, petri

117 plates were treated with different light regimes: 1$)$ immediately after being inoculated (0

118 days post-inoculation, 0dpi), 2) at 1 and/or 2dpi, after germination of spores begun, and 3)

119 at 4 dpi, when the mycelium of each strain was abundant. Temperature was maintained

120 constant at $20^{\circ} \mathrm{C}$, both during the light treatments and during fungal growth in the plates

121 under darkness, and monitored with an electronic datalogger. To evaluate how the light

122 treatments may affect fungal growth, colonies diameters were measured from the reverse

123 side in $\mathrm{cm}$ with a ruler in two directions along the time at $20^{\circ} \mathrm{C}$ for at least 7 days. For each

124 experimental condition, three colonies were evaluated. Control samples consisted of 125 inoculated plates maintained always under darkness at the same temperature. 
The percentage of growth inhibition was also calculated using the following formula: Percentage of growth inhibition $=100 \times(\mathrm{GC}-\mathrm{GSL}) / \mathrm{GC}$, where GC is growth of the control (continuous darkness) and GSL is the growth of the

129 fungal colony of the sample exposed to the light treatment (28).

131 Degree of germination: The degree of germination was determined in three replicate 132 samples using a Neubauer chamber. The number of germinated (already showing a germ tube) and ungerminated spores were counted and the degree of germination was expressed as the percentage of germinated spores. A total of 100 spores were counted in each replicate sample.

136

137 Blue light treatments: To test whether the effectiveness of blue light in reducing the 138 pathogens' growth infective capacities may depend on both light quantum flux and time of 139 exposure to light, inoculated plates were exposed to light quantum fluxes ranging from 30 140 to $700 \mu$ molm $^{-2} \mathrm{~s}^{-1}$ during periods ranging between $5 \mathrm{~min}$ and 3 days. The first experiment 141 was performed with the Pd1 strain, since $P$. digitatum is the major pathogen of citrus fruits 142 and this strain is highly resistant to the fungicides TBZ and imazalil. The matrix blue light 143 quantum flux/exposure time to light first ranged between 30 and $120 \mu \mathrm{molm}^{-2} \mathrm{~s}^{-1}$ and 144 between $5 \mathrm{~min}$ and $6 \mathrm{~h}$. On the basis of the results obtained, we further tested the effect of 145 applying $120 \mu \mathrm{molm}^{-2} \mathrm{~s}^{-1}$ for 6,24 and $72 \mathrm{~h}$ using the Pd1 and the PHI-41 strains, both 146 resistant to imazalil and TBZ. Moreover, we investigated the effect of the most effective 147 treatment on the infective capacity of the strain Pd1 when the light was applied at different 148 dpi $(0,1,2$ and 4$)$, and whether the effectiveness of the treatments was maintained after 149 removing the light stimulus. Finally, we studied whether light treatment duration may be 
150 reduced by sharply increasing quantum flux to $700 \mu \mathrm{molm}^{-2} \mathrm{~s}^{-1}$. Under such light regime,

151 the effectiveness of time periods equal or lower than $24 \mathrm{~h}$ was tested and the effect of the

152 selected treatment on fungal growth and morphology of the 4 strains was studied at 0 and 4

153 dpi.

154 Petri dishes were treated with the lid covers to avoid contaminations under the different

155 light regimes described above in Mammoth Pro dark growth tents $(60 \times 60 \times 160 \mathrm{~cm})$

156 (Mammoth Pro 60, Eltac Hidrofarm, Spain) equipped with velcro-sealable ventilation

157 panels $(300 \mathrm{~mm} \times 200 \mathrm{~mm})$ and tough fabric lined with $95 \%$ reflective mylar to assure

158 uniform light quantum flux. The light source was a LumiGrow Pro $650^{\mathrm{TM}}$ LED array

159 (LumiGrow, Novato, CA, USA) that emitted blue light at a center wavelength of $450 \mathrm{~nm}$

160 with a full width at half-maximum of $20 \mathrm{~nm}$. . Light quantum flux was measured and

161 adjusted using a spectroradiometer (GL Spectics, Sttutgart, Germany). The growth tents

162 had sufficient capacity for air exchange and were placed in a temperature and humidity

163 controlled room.

164

165 Statistical analysis: A mean comparison using the Tukey's test was performed to

166 determine if means values were significantly different $(\mathrm{P} \leq 0.05)$ between blue light-treated

167 and control samples (constant darkness) for each experimental light regime. The results

168 were the means of three replicated samples \pm S.E.M.

169

170 RESULTS AND DISCUSSION

171

172 Effect of LED blue light on growth of $\boldsymbol{P}$. digitatum strains resistant to fungicides 
173 Recently, it has been reported that LED blue light at a quantum flux of $40 \mu \mathrm{molm}^{-2} \mathrm{~s}^{-1}$

174 induces resistance against $P$. digitatum in citrus fruit (25) and also that blue light reduced

175 fungal development when $P$. digitatum culture was exposed to a higher quantum flux (120

$\left.176 \mu \mathrm{molm}^{-2} \mathrm{~s}^{-1}\right)(26)$. Therefore, we hypothesized that the effect of blue light inhibiting fungal

177 growth may increase with light quantum flux and that the duration of light treatment may

178 be shortened by increasing the quatum flux. Moreover, an interesting possibility would be

179 that blue light would even have a fungicidal effect that could be used in the packing-houses

180 as a mean of sanitation. This would be especially relevant since decay in citrus fruit is

181 mainly controlled by two fungicides and strains resistant to them are abundant. To test such

182 hypotheses, we first examined whether the growth of the P. digitatum Pd1 strain, resistant

183 to TBZ and imazalil, was affected by exposing the cultured plates from 5 min to $6 \mathrm{~h}$ to light

184 quantum fluxes ranging between 30 and $120 \mu \mathrm{molm}^{-2} \mathrm{~s}^{-1}$. Pd1 fungal growth was only

185 slightly reduced when the plates were treated with a quantum flux of $120 \mu \mathrm{molm}^{-2} \mathrm{~s}^{-1}$ for $6 \mathrm{~h}$

186 (see Supplementary Materials Fig. S1), and such reduction was not statistically significant.

187 Therefore, in a subsequent experiment, this light treatment was extended for $72 \mathrm{~h}$.

188 The effectiveness of the $120 \mu \mathrm{molm}^{-2} \mathrm{~s}^{-1}$ treatment applied for 6,24 and $72 \mathrm{~h}$ was

189 tested for Pd1 and also for PHI-41, which is another $P$. digitatum strain resistant to both

190 fungicides. Results confirmed that exposing the plates for $6 \mathrm{~h}$ to this quantum flux barely

191 reduced fungal growth of both pathogens and showed that the efficacy of the light treatment

192 may be increased by extending the time of exposure to blue light (Fig. 1). By day 7, the

193 efficacy of the $72 \mathrm{~h}$ treatment inhibiting fungal growth of the PHI-41 strain was c.a. 3 -fold

194 higher than that of the $24 \mathrm{~h}$ treatment (Fig. 1). Results also showed that differences afforded

195 by the $72 \mathrm{~h}$ treatment inhibiting fungal growth of the Pd1 and the PHI-41 strains were

196 initially low, and such differences were lost as time progressed (Fig. 1). Therefore, results 
197 indicate that susceptibility to blue light of strains from the same fungus genus may be

198 similar, while the effect of blue light varied when applying to different food relevant fungi

199 (22) or to various Penicillium fungal species that cause postharvest decay in citrus fruit

200 (26). Since the $72 \mathrm{~h}$ light treatment was only partially effective controlling fungal growth,

201 the question was whether increasing light exposure or quantum flux would be able to 202 control Penicillium growth and if such control might be even achieved if the light is applied 203 at different dpi.

Effect of delaying LED blue light application on growth and morphology of $P$. digitatum

207 Considering the points highlighted above, we first focused on studying the effect of exposing continuously, or for 3 days, the $P$. digitatum culture to the $120 \mu \mathrm{molm}^{-2} \mathrm{~s}^{-1}$ blue

209 light treatment at different dpi. The experiment was performed using the Pd1 strain. At 0 210 dpi, Pd1 fungal growth inhibition was about $45 \%$ after 3 days, when the plates were 211 continuously incubated under light (Fig. 2, 0 dpi). The diameter of the Petri plates used was 212 of $9 \mathrm{~cm}$. Therefore, this was the maximum fungal growth detected in the control plates 213 continuously maintained under darkness. This maximum was reached by day 11 in control 214 plates under dark conditions, but the experiment was prolonged to know whether this light 215 quantum flux may have a fungistatic effect although it was not possible to monitor growth 216 in control plates after that day. As shown in this graph (Fig.2, 0 dpi), by day 9, Pd1 fungal 217 growth was about 2-fold lower in the cultures held continuously under light than in those 218 transferred from light to darkness (non-continuous light). The above result reinforces the 219 idea that the efficacy of the treatment increases with its duration for the same light quantum 220 flux, and further suggests that $P$. digitatum fungal growth might be stalled by increasing 
221 exposure to blue light radiation for a given dose. Therefore, an interesting possibility is that 222 blue light might exert a fungistatic effect on $P$. digitatum if appropriate light quantum flux 223 is applied. This result is in concordance with data indicating that increasing radiation 224 intensity may in some cases maximize the benefits of UV-C on reducing decay caused by 225 Botrytis (20). However, mechanisms by which blue light may have a fungistatic effect on 226 Penicillium or other postharvest pathogens should be at least in part different. In this regard 227 it has to be considered that differences in sensitivity and responses of different fungi or 228 plants species to light of different wavelengths might be related to the different sensitivity 229 of light receptors $(22,29-31)$. The relevance of cryptochromes in the responses of plants 230 and fungi to blue light has been described $(31,33)$. However, to date it has not been shown 231 that cryptochromes can perceive UV-B or UV-C irradiations (34).

232 Delay in application of postharvest fungicides as well as of other alternative treatments 233 controlling decay caused by pathogenic fungi may alter their efficacy reducing both decay 234 incidence and in vitro growth $(9,35,36)$. Therefore, we also investigated whether delaying 235 LED blue light application may influence the growth of the fungus. Results revealed the 236 potential of blue light for reducing $P$. digitatum fungal growth if the cultured plates are 237 light-treated at different time points after pre-inoculation with the fungus since blue light $238\left(120 \mu \mathrm{molm}^{-2} \mathrm{~s}^{-1}\right)$ was able to restrict growth of Pd1 P.digitatum strain regardless of time of 239 light treatment delay (Fig 2). Fungal growth was almost stopped always after 8 days of 240 continuous light application and was c.a. the $50 \%$ that of the colonies held under darkness. 241 These results indicate that the $120 \mu^{m o l m}{ }^{-2} \mathrm{~s}^{-1}$ light quantum flux did not avoid spore 242 germination although it may stall fungal growth. Moreover, it is interesting to note that the 243 time required to achieve such effect when applying continuously this light quantum flux 244 barely depended on the developmental stage of the fungus. 
Results further showed that if the colonies treated at $0,1,2$ or 4 dpi for 3 days with a $120 \mu \mathrm{molm}^{-2} \mathrm{~s}^{-1}$ blue light quantum flux were shifted to dark before reaching the stalling

247 growth they still grew and that, after light removal, the fungal growth rate depended on the 248 day in which the light was applied (Fig. 2). Thus, the slopes found for $0,1,2$, and 4 dpi were $0.859,0.802,0.630$ and 0.2418 , respectively. Such slopes were calculated from the day it finished the light treatment for each dpi trial and during the period the growth was still lineal. These results indicate that the loss of light efficacy inhibiting fungal growth when the cultures were transferred to darkness was faster at 0 dpi and lower at 4 dpi, which suggests that the already grown mycelium losses the ability to cope with the damage 254 inflicted by the light treatment.

255 We further examined whether the $120 \mu \mathrm{molm}^{-2} \mathrm{~s}^{-1}$ blue light quantum flux was able to 256 stop the colonies' growth if they were shifted to the dark after reaching the stalling growth. 257 This experiment was performed at 0 and at 4 dpi since these two post-inoculation periods 258 showed the highest difference in growth rate when the plates were transferred to darkness 259 after the 3 days light treatment. Moreover, the incubation period was considerably 260 extended. Results confirmed the behavior found in the previous experiment since 261 continuous light induced a delayed fungistatic effect after 8 days of fungal growthand this 262 effect was maintained until the end of the experiment (22 days) (Fig. 3). Furthermore, the 263 loss of light efficacy of the 3 days light treatment was faster at 0 dpi, (Fig. 3). Results also 264 showed that no further growth occurred if the colonies treated with light at 0 or 4 dpi were 265 transferred to darkness after the mycelium growth was stalled (Fig. 3). That is, the efficacy 266 of the treatment was not reversed after removing the light stimulus.

267 Morphology of Pd1 P. digitatum strain grown under different light regimes is shown in Fig. 268 S2 (see Supplementary Materials) and Fig. 4. In the 0 dpi trial, slight changes in the 
morphology of the colonies occurred when this light quantum flux was applied for 3 days and then the plates were transferred to dark, but extending the light exposure period clearly affected the mycelium. Sporulation was already observed by day 4 in control plates held under darkness (Fig. S2a). In contrast, it was not observed in the cultures treated for 3 days with light and then transferred to darkness for 1 additional day (Fig. S2b) or in cultures held under continuous light (Fig. S2c). By day 7, the colonies held under darkness showed the characteristic green conidia and white mycelium of $P$. digitatum (Fig. 4a). The morphology of the cultures exposed to non-continuous light (Fig. 4b and c) was similar, although the 3 days light treatment delayed the fungal growth (Fig. 3). These results indicate that noncontinuous light ( 3 days light) at a quantum flux of $120 \mu \mathrm{molm}^{-2} \mathrm{~s}^{-1}$ has only a slight effect retarding growth and sporulation when the light is applied at 0 dpi. However, extending light exposure (continuous light) had a noticeable effect (Fig 4d-f). By 7 days, the presence of brownished mycelium was already observed, although white mycelium still predominated (Fig. 4d). At day 11 under continuous light, the older part of the colony was mostly brown (Fig. 4e) but the remaining white mycelium was still able to sporulate as 284 green conidia were very evident after shifting the plates to darkness for 4 days (Fig. 4f). 285 Overall results indicate then that exposing P.digitatum to a $120 \mu \mathrm{molm}^{-2} \mathrm{~s}^{-1}$ blue light for a 286 short period (3 days) at 0 dpi slightly affects growth and sporulation and, therefore, the 287 effect of the treatment on reducing fungal growth may be reversed after removing the light. 288 However, longer light exposure (11 days) may have a dramatic effect on fungal viability, 289 which may explain, at least in part, the lack of reversibility of the effect of the 11 days light 290 treatment on fungal growth after shifting the plates from light to darkness.

291 Examination of changes induced in morphology by continuous and non-continuous 292 light at 4 dpi (Fig. 4g-1) in cultures treated with the same light quantum flux further 
293 supported that older mycelium was more sensitive to this light quantum flux. Treating $P$.

294 digitatum with light for 3 days at 4 dpi caused brownish on the center and older part of the 295 colony (Fig. 4g), a fact not found at 0 dpi. Thus, by 7 days of fungal growth, colony 296 appearance was very different depending on when the 3 days light treatment was applied, 297 either at 0 or 4 dpi (Fig. 4b and g). This differential effect was maintained after further 298 incubation in the dark (Fig. 4c and 4h). As shown in Fig. 4i, further incubation in the dark 299 allowed the outer younger part of the colony treated at 4 dpi with light for 3 days to 300 sporulate, however, colony growth was stalled (Fig. 3). Therefore, the 3 days light 301 treatment did not completely avoided sporulation although it had an important inhibitory 302 effect on fungal growth when it was applied at 4 dpi (Fig. 3). In contrast, it barely affected 303 the fungus morphology and had only a transient inhibitory effect when it was applied at 0 304 dpi (Fig. 3). The multiple effects of UV treatments both in pathogens and fruits have been 305 widely studied $(10,37,38)$. Information related to blue light-emitting diodes induced 306 responses against pathogens causing diseases in fruits is less abundant (39). As far as we 307 know, there is only one report related to the study of the mechanisms of blue light effects 308 on $P$. digitatum. This study showed that low blue light quantum flux $\left(40 \mu \mathrm{molm}^{-2} \mathrm{~s}^{-1}\right)$ 309 reduces the activity of the pectolytic enzyme polygalacturonase produced by the fungus 310 (26).

311 Changes in morphology found when continuous light was applied further support the 312 higher susceptibility of the fungus to light at 4 dpi as compared to 0 dpi. As shown in Fig $3134 \mathrm{j}-1$, applying the light for 7 days at 4 dpi led to abundant brownish mycelium and 314 completely inhibited sporulation. This effect was not reversed after shifting the plates to 315 darkness. This might indicate severe hyphae damage or even cell death. In this regard, it 316 should be noted the participation of oxidative stress in cell death and that the accumulation 
317 of singlet oxygen is involved in the inhibitory effect of $450 \mathrm{~nm}$ blue light generated by LED 318 on growth of $B$. cinerea (40). Therefore, focusing on oxidative stress would be an 319 interesting approach to decipher the mode of action of blue light inhibiting $P$. digitatum 320 growth. Likewise, it should be considered that blue light-mediated damage to bacteria cells 321 has been related to the development of vacuoles within the cytoplasm, implying that 322 damage was related to intracellular chromophores excited by the blue light, to the release of 323 cytoplasmatic material to the surrounding environment and to cytoplasmic disruption (41)

\section{Fungicidal effect of blue light on Penicillium digitatum strains resistant and sensitive} to fungicides and on Penicillium italicum

327 The above results suggest that blue light might reach a fungicidal effect on P.digitatum if 328 the quantum flux is substantially increased and also that increasing the quantum flux might 329 favor a drastic shortening of the treatment. If these were the cases, then blue light might be 330 a useful tool for sanitation or for reducing contaminations even if it is applied after spore 331 germination. These ideas were tested by increasing c.a. 6-fold the light quantum flux (700 $\left.332 \mu \mathrm{molm}^{-2} \mathrm{~s}^{-1}\right)$. The effect of this light dose was assayed in four different strains, three of them 333 from P.digitatum (Pd1, PHI-41 and PHI-26) and one from P. Italicum (PHI-1). Two of the 334 P.digitatum strains (Pd1 and PHI-41) are resistant to the fungicides imazalil and TBZ.

335 The effect of shortening the $700 \mu$ molm $^{-2} \mathrm{~s}^{-1}$ light treatment was first investigated in the 336 PHI-41 (P. digitatum) and PHI-1 (P. italicum) strains. Results show that the effectiveness 337 of this high light quantum flux also increases with duration of the treatment and that 338 applying the light for $18 \mathrm{~h}$ had already a fungicidal effect on both strains because the fungi 339 did not resume growing after the plates were returned to darkness (Fig. 5). After this light 340 treatment, the percentage of germination was $0 \%$, while c.a. $81 \%$ of the spores germinated 
341 in the control plates held under darkness. Reducing time of light application to $3 \mathrm{~h}$ had no 342 inhibitory effect on PHI-41 growth and slightly inhibited the growth of PHI-1 (data not 343 shown).

344 In a subsequent experiment, we extended the incubation period of the inoculated plates 345 and further confirmed that applying $700 \mu \mathrm{molm}^{-2} \mathrm{~s}^{-1}$ blue light for $18 \mathrm{~h}$ has a fungicidal 346 effect on all the studied strains if the light is applied at 0 dpi (Fig. 6). Growth of these 347 strains was also reduced when this treatment was applied at 4 dpi (Fig. 6). Therefore, it 348 appears that the fungal spores are more susceptible to the higher quantum flux doses but 349 this different effect is not observed with 4 days old hyphae. Applying $700 \mu \mathrm{molm}^{-2} \mathrm{~s}^{-1}$ blue 350 light for $18 \mathrm{~h}$ affected the mycelium of the 4 strains grown for 4 days. Changes in 351 morphology of the PHI-26 strain grown for 4 days at $20{ }^{\circ} \mathrm{C}$ and then exposed to this 352 treatment are shown in Fig. 7. Patterns of changes in the rest of strains assayed were similar 353 (not shown). Brownish

354 areas were already present after finishing the treatment (day 5) and also 2 days after 355 transferring the cultures to darkness (day 7), although the white mycelia predominated and 356 was able to further sporulate when the light was applied at 4 dpi for just $18 \mathrm{~h}$. This would 357 explain further fungal growth (Fig. 6 and 7) when this treatment was applied. As shown 358 above, continuous light had a clear and not reversible detrimental effect on fungal growth 359 even though it was applied at a lower quantum flux (Fig 4). Therefore, global results 360 indicate that fungal spores cannot germinate under short high light quantum flux ( $0 \mathrm{dpi})$ and 361 that a continuous lower light quantum flux is able to suppress both sporulation and 362 mycelium growth when applied to already grown mycelium (4 dpi). Since blue light may 363 avoid sporulation of Penicillium strains sensitive and resistant to the fungicides used to 364 reduce disease incidence in citrus fruits, this new strategy might constitute a useful 
365 alternative method for sanitation within integrated control programs for postharvest pest 366 management of citrus fruits. Blue light would be of interest to reduce the use of fungicides 367 by reducing contaminations of the fungicide solutions, which might allow reducing the 368 concentrations applied or, at least, further re-utilization of such solutions. Although these 369 solutions may be turbid, it is noticing the development of light processing methods for 370 highly turbid fluids (42). Moreover, blue light could be used as a mean to minimize 371 contaminations in cold storage rooms and other strategic points in the packing-houses. This 372 would be especially relevant since decay in citrus fruit is mainly controlled by two 373 fungicides (imazalil and TBZ) with different mode of action, whose use must be rotated in 374 the packing- and store-houses for avoiding the development of strains resistant to them. 375 Therefore, this study encourages further research simulating industrial conditions to 376 implement blue light within integrated control programs for pest management of citrus 377 fruits. Results indicate that the development of technologies able to induce high quantum 378 fluxes of blue light would be helpful to shorten the light treatments. This study was limited 379 by the light output of the LED array used but development of systems that use high-power 380 LED arrays / light sources could enable the delivery of very high LED blue light doses for 381 decontamination applications. This innovative emerging technology could be useful to the 382 industry to reduce citrus fruit losses, and probably of other fruit and vegetable crops, caused 383 by pathogens sensitive and resistant to fungicides. Moreover, it could help to open new 384 markets demanding fruits non-treated with fungicides, while minimizing energy cost and 385 toxic residues of fungicides

\section{CONCLUSIONS}


388 Results showed the potential of LED blue light for controlling the growth of $P$. digitatum 389 and $P$. italicum, which are the major pathogens of citrus fruits after harvesting, and that 390 efficacy increases with the duration of the treatment and with the light quantum flux. The 391 older mycelium was more sensitive to blue light. Spore germination of strains sensitive and 392 resistant to fungicides can be completely avoided by exposing them for a short period of 393 time to high blue light quantum flux $\left(700 \mu \mathrm{molm}^{-2} \mathrm{~s}^{-1}\right)$. Lower quantum fluxes $\left(120 \mu \mathrm{molm}^{-}\right.$

$\left.394{ }^{2} \mathrm{~s}^{-1}\right)$ have a fungicidal effect when continuously applied to already grown mycelium (4 dpi).

395 Therefore, the combination of high quantum flux followed by a continuous lower quantum 396 flux may reduce both sporulation and mycelium viability. Global results indicate that blue 397 light may be a tool to reduce contaminations and infections caused by $P$. digitatum and $P$. 398 italicum strains in postharvest facilities by reducing pathogen inoculum.

400 ACKNOWLEDGEMENTS: Special thanks are due to Drs L. González-Candelas and 401 A.R. Ballester for providing the Penicillium strains and also to Dr L. González-Candelas 402 for critically revising the manuscript. The technical assistance of L. Collazo and A. 403 Izquierdo is gratefully acknowledged. This work was supported by research grants 404 AGL2013-41734-R from the Comisión Interministerial de Ciencia y Tecnología (CICYT), 405 Spain, and by PROMETEOII/2014/027 from the Generalitat Valenciana, Spain. Dr. F. 406 Alférez acknowledges a Ramón y Cajal Contract (Spanish Government and Fondo Social 407 Europeo).

\section{SUPPLEMENTARY MATERIALS}

410 Additional Supplementary Information may be found in the online version of this article: 
411 Figure S1. Growth of the Pd1 P. digitatum strain in potato-dextrose agar (PDA) 412 after being treated from 5 min to 360 min with blue light at a quantum flux of $120 \mu \mathrm{molm}^{-}$ $413{ }^{2} \mathrm{~s}^{-1}$. Results represent the means of three replicates samples \pm S.E.M. No significant 414 differences $(\mathrm{P}<0.05)$ were found between the control and any of the light-treated samples 415 for the same storage period.

416 Figure S2. Morphology of $P$. digitatum in response to the $120 \mu \mathrm{molm}^{-2} \mathrm{~s}^{-1}$ LED blue 417 light treatment. The fungus was grown in PDA at $20{ }^{\circ} \mathrm{C}$ for 4 days and photographed: a) 4 418 days darkness (D, control), b) 3 days light (L) plus 1 day darkness (D), c $=4$ days light (L). 419 420 


\section{REFERENCES}

422 1. Sánchez-Torres, P. and J. J. Tuset (2011) Molecular insights into fungicide resistance 423 in sensitive and resistant Penicillium digitatum strains infecting citrus. Postharvest Biol. 424 Technol. 59,159-165.

425 2. Nigro, F., A. Ippolito and G. Lima (1998) Use of UV-C light to reduce Botrystis 426 storage rot of table grapes. Postharvest Biol. Technol. 13, 171-181.

427 3. Droby, S., M. Wisniewski, D. Macarisin and C. Wilson (2009). Twenty years of 428 postharvest biocontrol research: is it time for a new paradigm? Postharvest Biol. Technol. $42952,137-145$.

430 4. Sharma, R. R., D. Singh, and R. Singh (2009) Biological control of postharvest 431 diseases of fruits and vegetables by microbial antagonists: a review. Biol. Control 50, 205432221.

433 5. Romanazzi, G., A. Lichter, F. M. Gabler and J. L. Smilanick (2012) Recent advances 434 on the use of natural and safe alternatives to conventional methods to control postharvest 435 grey mold of table grapes. Postharvest Biol. Technol. 63, 141-147.

436 6. Ballester, A. R., M. T. Lafuente, C. H. R. De Vos, A. Bovy and L. González-Candelas 437 (2013) Citrus phenylpropanoids and defence against pathogens. Part I: Metabolic profiling in 438 elicited fruits. Food Chem. 136, 178-185.

439 7. Montesinos-Herrero, C., J. L. Smilanick, J. S. Tebbets, S. Walse and L. Palou (2011) 440 Control of citrus postharvest decay by ammonia gas fumigation and its influence on the 441 efficacy of the fungicide imazalil. Postharvest Biol. Technol. 59, 85-93.

442 8. Ballester, A. R., M. T. Lafuente and L. González-Candelas (2006) Spatial study of 443 antioxidant enzymes, peroxidase and phenylalanine ammonia-lyase in the citrus fruit444 Penicillium digitatum interaction. Postharvest Biol. Technol. 39, 115-124. 
445 9. Eckert, J. W. and N. F. Sommer (1967) Control of diseases of fruits and vegetables by 446 postharvest treatment. Annu. Rev. Phytopathol. 5, 391-432.

447 10. Droby, S., E. Chalutz, B. Horev, L. Cohen, V. Gaba, C. L. Wilson and M. Wisniewski 448 (1993) Factors affecting UV-induced resistance in grapefruit against the green mould decay 449 caused by Penicillium digitatum. Plant Pathol. 42, 418-424.

450 11. Smilanick, J.L., M. F. Mansour and D. Sorenson (2006) Pre- and postharvest 451 treatments to control green mold of citrus fruit during ethylene degreening. Plant Dis. 90, $452 \quad 89-96$.

453 12. Schirra, M., S. D'Aquino, A. Palma, A. Angioni and P. Cabras (2008) Factors 454 affecting the synergy of thiabendazole, sodium bicarbonate, and heat to control postharvest 455 green mold of citrus fruit. J. Agric. Food Chem. 56, 10793-10798.

456 13. Palou, L., J. L. Smilanick, and S. Droby (2008) Alternatives to conventional 457 fungicides for the control of citrus postharvest green and blue moulds. Stewart Postharvest. 458 Rev. 2, 1-16.

459 14. Malik, Z., J. Hanania and Y. Nitzan (1990) New trends in photobiology bactericidal 460 effects of photoactivated porphyrins-An alternative approach to antimicrobial drugs. 461 J. Photochem. Photobiol. B: Biol. 5, 281-293.

462 15. Papageorgiou, P., A. Katasambas and A. Chu (2000) Phototherapy with blue (415 $463 \mathrm{~nm})$ and red $(660 \mathrm{~nm})$ light in the treatment of acne vulgaris. Br. J. Dermatol. 142, 973464978.

465 16. Ashkenazi, H., Z. Malik, Y. Harth and Y. Nitzan (2003) Eradication of 466 propionibacterium acnes by its endogenic porphyrins after illumination with high 467 intensity blue light. FEMS Immunol. Med. Microbiol. 35, 17-24.

468 17. Lipovsky, A., Y. Nitzan, H. Friedmann and R. Lubart (2009) Sensitivity of 
469

470

471

472

473

474

475

476

477

478

479

480

481

482

483

484

485

486

487

488

489

490

491

492

Staphylococcus aureus strains to broadband visible light. J. Photochem. Photobiol. 85, 255260.

18. E. Endarko, M. Maclean, I. V. Timoshkin, S. J. MacGregor and J. G. Anderson (2012) High-Intensity $405 \mathrm{~nm}$ Light Inactivation of Listeria monocytogenes. Photochem. Photobiol. 88: 1280-1286.

19. D'hallewin, G., M. Schirra, E. Manueddu, A. Piga and S. Ben Yehoshua (1999) Scoparone and scopoletin accumulation and ultraviolet-C induced resistance to postharvest decay in oranges as influenced by harvest date. J. Am. Soc. Hortic. Sci. 124, 702-707.

20. Cote, S., L., Rodoni, E. Miceli, A. Concellón, P. M. Civello and A. R. Vicente (2013) Effect of radiation intensity on the outcome of postharvest UV-C treatments. Postharvest Biol.Technol. 83, 83-89.

21. Folta K. and K. S. Childers (2008). Light as a growth regulator: controlling plant biology with narrow-bandwidth solid-state lighting systems. Hortsci. 43, 1957-1964

22. Schmidt-Heydt, M., C. Rüfer, F. Raupp, A. Bruchmann, G. Perrone and R. Geisen (2011) Influence of light on food relevant fungi with emphasis on ochratoxin producing species. Int. J. Food Microbiol. 145, 229-237.

23. Ondrusch, N. and J. Kreft (2011) Blue and red light modulates SigB-dependent gene transcription, swimming motility and invasiveness in Listeria monocytogene. PLoS ONE 6(1): e16151. doi:10.1371/journal.pone.0016151

24. Nguyen, T. P., M. D. Danyluk and K. R. Schneider (2013) Growth, reduction, and survival of bacteria on melon types. University of Florida IFAS Extension. FSHN12-07, 145.

25. Alférez, F., H-L. Liao and J. K. Burns (2012) Blue light alters infection by Penicillium digitatum in tangerines. Postharvest Biol. Technol. 63, 11-15. 
493 26. Liao, H-L., F. Alférez and J. K. Burns (2013). Assessment of blue light treatments on 494 citrus postharvest diseases. Postharvest Biol. Technol. 81, 81-88.

495 27. Adaskaveg, J.E., H. Föster and N. F. Sommer (2002) Principles of postharvest 496 pathology and management of decay of edible horticultural crops. In Postharvest 497 Technology of Horticultural Crops: (Edited by A. A. Kader, Third edition), pp. 163-195. 498 Editorial University of California Agriculture and Natural Resources Publication 3311.

499 28. Fadda, A., A. Barberis, S. D’Aquino, A. Palma, A. Angioni, F. Lai, and M. Schirra 500 (2015). Residue levels and performance of potassium sorbate and thiabendazole and their 501 co-application against blue mould of apples when applied as water dip treatments at 20 or $50253^{\circ} \mathrm{C}$. Postharvest Biol. Technol. 106, 33-43.

503 29. Ensminger, P.A. and E. Schäfer (1992) Blue and ultraviolet-B light photoreceptors in 504 parsley cells. Photochem. Photobiol. 55, 437-447.

505 30. Losi, A. and W. Gärtner (2011) Old chromophores, new photoactivation paradigms, 506 trendy applications: Flavins in blue light-sensing photoreceptors. Photochem. Photobiol. $50787,491-510$.

508 31. Heintzen, Ch. (2012) Plant and fungal photopigments. WIREs Membr. Transp. 509 Signal. 1, 411-432. Doi:10.1002/wmts.36.

510 32. Chico, J. M., G. Fernández-Barbero, A. Chini, P. Fernández-Calvo, M. Díez-Díaz and 511 R. Solano (2014) Repression of jasmonate-dependent defenses by shade involves 512 differential regulation of protein stability of MYC transcription factors and their JAZ 513 repressors in Arabidopsis. Plant Cell 26, 1967-1980.

514 33. Heijde, M. and R. Hulm (2012) UV-B photoreceptor-mediated signalling in plants. 515 Trends Plant Sci. 17, 230-237. 
516 34. Wade, H. K., T. N. Bibikova, W. J. Valentine and G. I. Jenkins (2001) Interactions

517 within a network of phytochrome, crytochrome and UV-B phototransduction pathways 518 regulate chalcone synthase gene expression in Arabidopsis leaf tissue. Plant J. 25, 675-685.

519 35. Sugar, D. and S. R. Basile (2011) Orchard calcium and fungicide treatments mitigate 520 effects of delayed postharvest fungicide applications for control of postharvest decay of 521 pear fruit. Postharvest Biol. Technol.60, 52-56.

522 36. Gatto, M. A., A. Ippolito, V. Linsalata, N. A. Cascarano, F. Nigro, S. Vanadia and D. 523 Di Venere (2011) Activity of extracts from wild edible herbs against postharvest fungal 524 diseases of fruit and vegetables. Postharvest Biol. Technol. 61, 72-82.

525 37. Asad, L. M. B. O., A. A. de Carvalho, I. Felzenszwalb, A. C. Leitao and N. R. Asad 526 (2000) $\mathrm{H}_{2} \mathrm{O}_{2}$-induced cross-protection against UV-C killing in Escherichia coli is blocked 527 in a lexA (Def) background. J. Phytochem. Photobiol. B Biol. 54, 67-71.

528 38. Zlatev, Z., F. J. C Slidon and M. Kaimakanova (2012) Plant physiological responses 529 to UV-B radiation. Emir. J. Food Agric. 24, 481-501.

530 39. Kim, K., H-S. Kook, Y-J. Jang, W-H. Lee, S. Kama-Kannan, J-J. Chae and K-J. Lee 531 (2013) The effect of blue-light-emitting diodes on antioxidant properties and resistance to 532 Botrytis cinerea in tomato. J. Plant Pathol. Microb. 4:203.doi:10.4172/2157-7471.1000203.

533 40. Imada, K., S. Tanaka, Y. Ibaraki, K. Yoshimura and S. Ito (2014) Antifungal effect of 534 405-nm light on Botrytis cinera. Letters Appl. Microbiol. 59, 670-676.

535 41. Dai, T., A. Gupta, Y-Y. Huang, R. Yin, C. K. Murray, M. S. Vrahas, M. E. Sherwood, 536 G. P. Tegos, M. R. Hamblin (2013) Blue light rescues mice from potentially fatal 537 Pseudomonas aeruginosa burn infection: efficacy, safety and mechanism of action. 538 Antimicrob. Agents Chemother. 57, 1238-1245. 
539 42. Huan, Y., R. Sido, R. Huang and H. Chen (2015) Application of water-assisted pulsed 540 light treatment to decontaminate raspberries and blueberries from Salmonella. Int. J. Food 541 Microb. 208, 43-50.

542

543

544 


\section{Figure legends}

546 Figure 1. Effect of treating PHI-41 and Pd1 P. digitatum strains with $120 \mu \mathrm{molm}^{-2} \mathrm{~s}^{-1} \mathrm{LED}$ 547 blue light for $6(\circ), 24(\square)$ and $72 \mathrm{~h}(\nabla)$ on fungal growth and on fungal growth inhibition.

548 Control samples were always maintained under darkness $(\bullet)$. The treatments were applied 549 immediately after inoculating ( 0 dpi) the plates with $5 \mu 1$ of $10^{6}$ conidia $\mathrm{ml}^{-1}$. Results 550 represent the means of three replicates samples \pm S.E.M. Significant differences $(P<0.05)$ 551 between the light-treated PHI-41 and Pd1 strains and their respective controls continuously 552 held under darkness for the same storage period were found from day 3 when the samples 553 were treated with light for $72 \mathrm{~h}$. Significant differences were only maintained till day 5 554 when the light was applied for $24 \mathrm{~h}$, and no statistic difference was found when it was 555 applied for $6 \mathrm{~h}$.

557 Figure 2. Effect of treating the Pd1 P. digitatum strain with $120 \mu \mathrm{molm}^{-2} \mathrm{~s}^{-1}$ LED blue light 558 at different dpi on fungal growth. The cultures were treated continuously with this light 559 quantum flux $(\bigcirc)$, or for 3 days and then shifted to darkness (non-continuous light, $\square$ ).

560 Control samples were always held under darkness $(\bullet)$. The arrows indicate when the light 561 treatments started $\left(0,1,2\right.$ and 4 dpi). All the samples were inoculated with $5 \mu 1$ of $10^{6}$ 562 conidia $\mathrm{ml}^{-1}$. Results in the upper panels represent the means of three replicate samples \pm 563 S.E.M and the asterisks indicate significant differences $(\mathrm{P}<0.05)$ between the treated and 564 the control samples for the same storage period. The statistical analysis was not performed 565 for the 14 days samples since the diameter of the control colony held under darkness was 566 close or higher than $9 \mathrm{~cm}$. Data missing in each plot corresponding to the day 14 were close 567 or higher than $9 \mathrm{~cm}$. Data in the lower panels show the rate of growth when the cultures 568 were treated with non-continuous light at different dpi. 
569

570

571

572

573

574

Figure 3. Effect of treating the Pd1 $P$. digitatum strain with $120 \mu \mathrm{molm}^{-2} \mathrm{~s}^{-1} \mathrm{LED}$ blue light at 0 and 4 dpi on fungal growth. The cultures were treated continuously with this light (L) quantum flux (O), or for 3 days $(\square)$ and then shifted to darkness (D), in each dpi trial. Other sets of samples for each dpi were treated with light for 11 days, to reach the mycelium growth stalling, and then shifted to darkness $(\triangle)$. Control samples were always maintained under darkness $(\bullet)$. The arrows indicate when the light treatments started or finished ( 0 and $4 \mathrm{dpi})$. The $\mathrm{L}$ in the arrows indicates treatment with continuous light, while LD3 and LD11 indicate the days the plates were shifted to darkness after being treated for 3 and 11 days with light, respectively. All the samples were inoculated with $5 \mu 1$ of $10^{6}$ conidia $\mathrm{ml}^{-1}$. Results represent the means of three replicates samples \pm S.E.M. Significant differences $(\mathrm{P}<0.05)$ between the treated and the control samples for the same storage period were found from day 3 at $0 \mathrm{dpi}$ and from day 8 at 4 dpi. The diameter of the control plates (darkness, D) was close or higher than $9 \mathrm{~cm}$ after day 11 and, therefore, these values were not included in the plots.

Figure 4. Changes in the morphology of $P$. digitatum during its growth under different light regimes. The quantum flux of the LED blue light was $120 \mu \mathrm{molm}^{-2} \mathrm{~s}^{-1}$ light, the light was applied both at 0 (a-f) and at 4 dpi (g-l), and the fungus was grown in PDA at $20^{\circ} \mathrm{C}$ and photographed at different time points. In the photographs, $d$ indicates the number of days, D darkness and L light. The symbol + indicates the shifting from light to darkness or from darkness to light.

Figure 5. Effect of treating the PHI-41 (P. digitatum) and PHI-1 (P. italicum) strains with $700 \mu$ molm $^{-2} \mathrm{~s}^{-1}$ LED blue light for $6(\diamond), 9(\bigcirc), 18(\nabla)$ or $24 \mathrm{~h}(\square)$ on fungal growth and 
593 fungal growth inhibition. Control samples were always held under darkness (•). The 594 treatments were applied immediately after inoculating $(0 \mathrm{dpi})$ the plates with $5 \mu 1$ of $10^{6}$ 595 conidia $\mathrm{ml}^{-1}$. Results represent the means of three replicates samples \pm S.E.M. Significant 596 differences $(\mathrm{P}<0.05)$ between the control and the samples subjected to any treatment for 597 the same storage period were found from day 2.

598

599 Figure 6. Effect of treating the Pd1, PHI-26, PHI-41 $P$. digitatum strains and the PHI-1 $P$. 600 italicum strain with $700 \mu \mathrm{molm}^{-2} \mathrm{~s}^{-1}$ LED blue light for $18 \mathrm{~h}$ at $0(\mathrm{O})$ and $4 \mathrm{dpi}(\square)$ on 601 fungal growth. Control samples were always held under darkness $(\bullet)$. All the plates were 602 inoculated with $5 \mu 1$ of $10^{6}$ conidia $\mathrm{ml}^{-1}$. Results represent the means of three replicated 603 samples \pm S.E.M. Significant differences $(\mathrm{P}<0.05)$ between the control and the samples 604 subjected to the light treatment at 0 and 4 dpi were found along the whole experiment for 605 the same storage period. Data missing in each darkness plot or in the plot corresponding to 606 the PHI-26 culture treated with light at 4 dpi were equal or higher than $9 \mathrm{~cm}$.

607

608 Figure 7. Changes in the morphology of the PHI-26 P. digitatum strain during growth at 20

$609{ }^{\circ} \mathrm{C}$. Cultures were held continuously under darkness (D) (pictures in upper panels), or were 610 treated for $18 \mathrm{~h}$ with $700 \mu \mathrm{molm}^{-2} \mathrm{~s}^{-1}$ LED blue light (L) at day 4 (4 dpi) and then shifted for 611 different number of days (d) to darkness. This light regime avoided sporulation at 0 dpi and 612 therefore, no photograph corresponding to 0 dpi is shown. Pattern of changes in the rest of 613 strains assayed was very similar and, therefore, only the photographs corresponding to the 614 PHI-26 strain are shown to better visualize the effect of blue light. 
Fig. 1

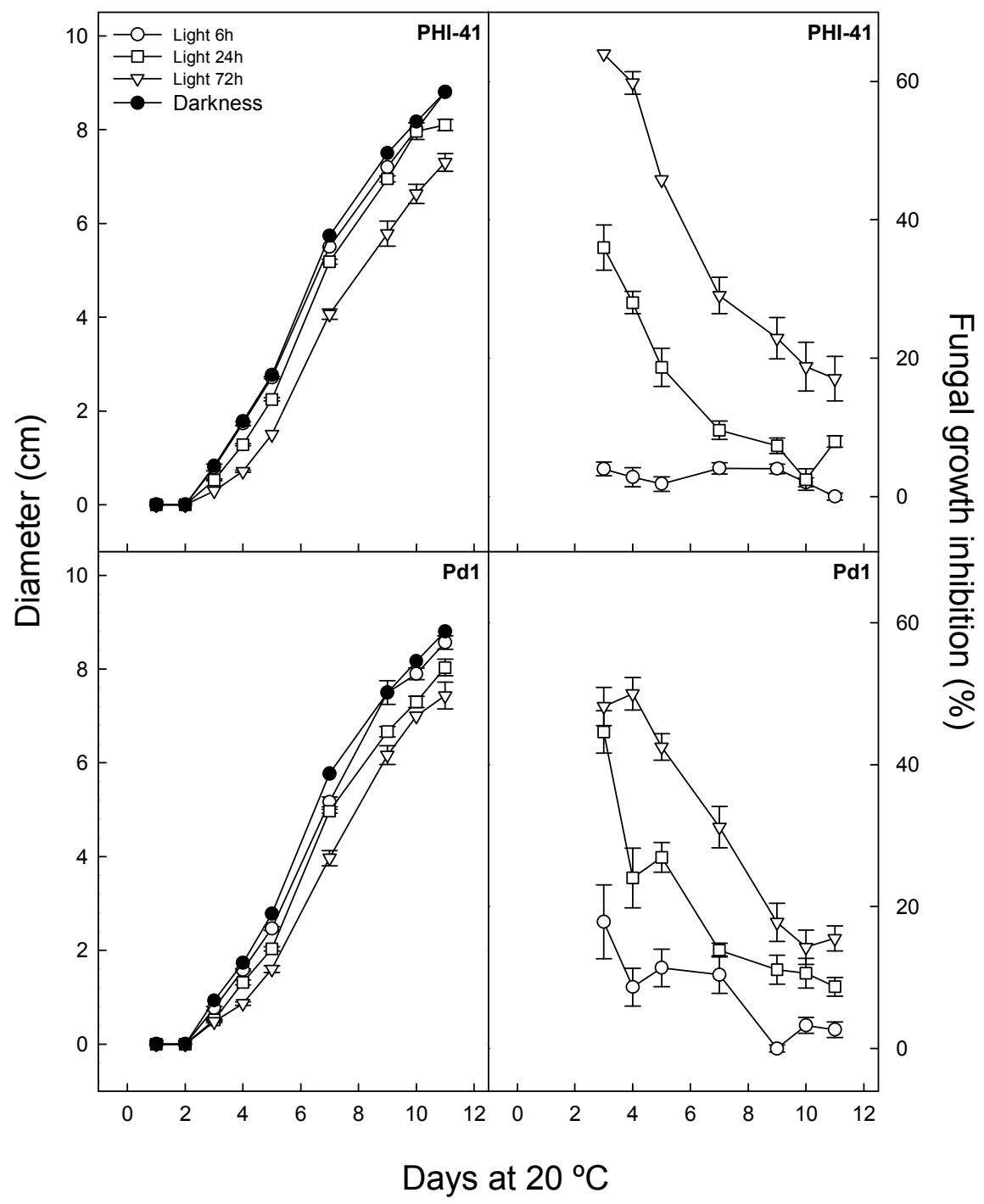


Figure 2

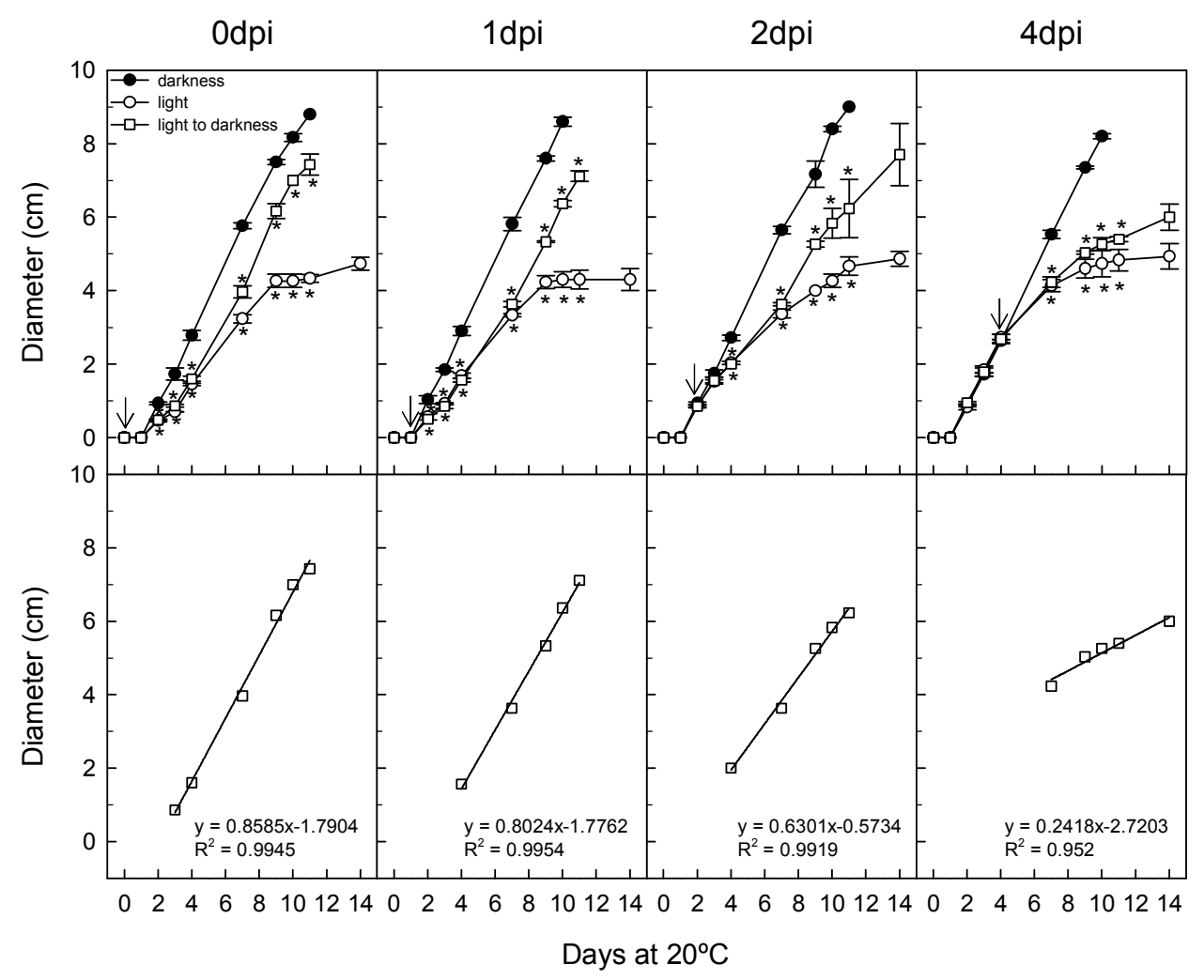


Figure 3

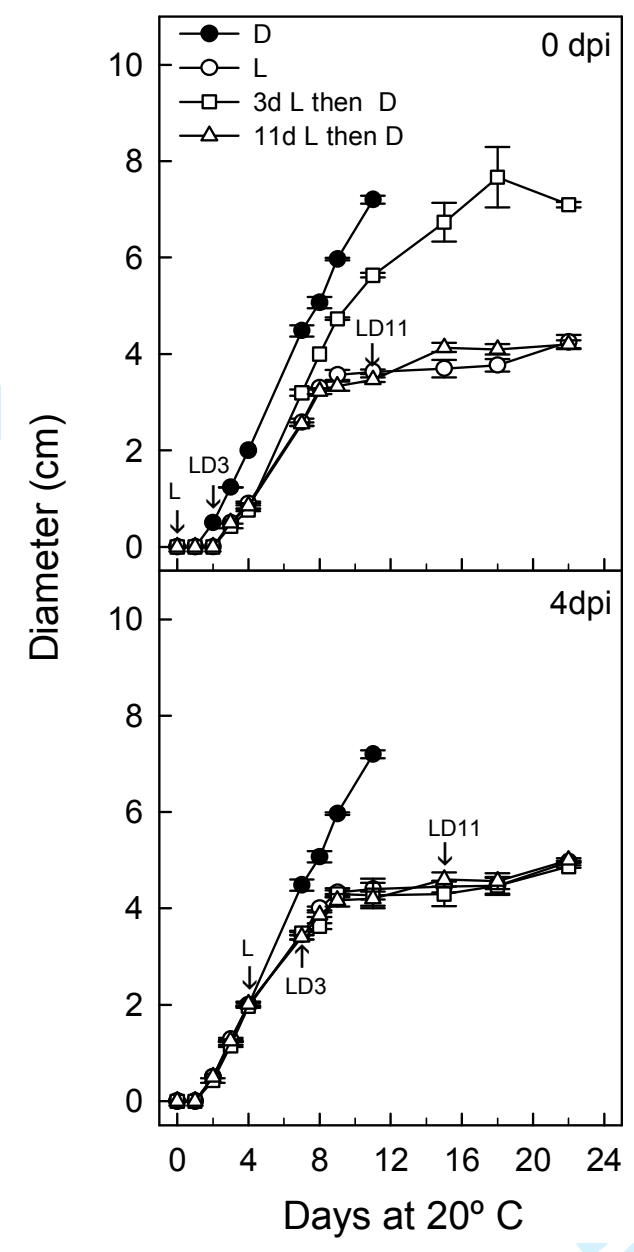


FIG 4

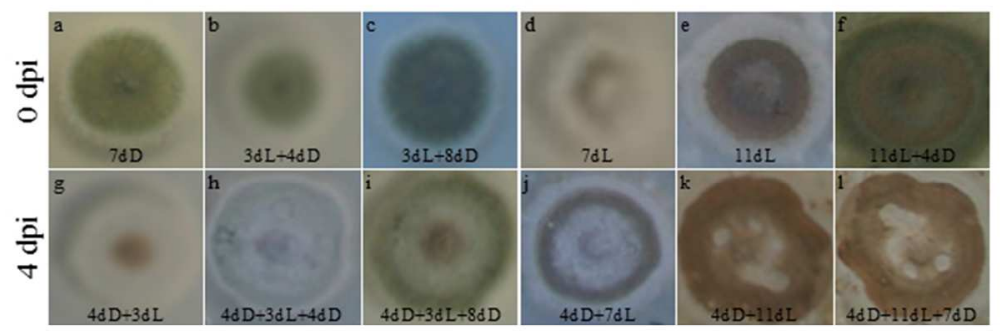

$190 \times 254 \mathrm{~mm}(96 \times 96 \mathrm{DPI})$ 
Fig. 5

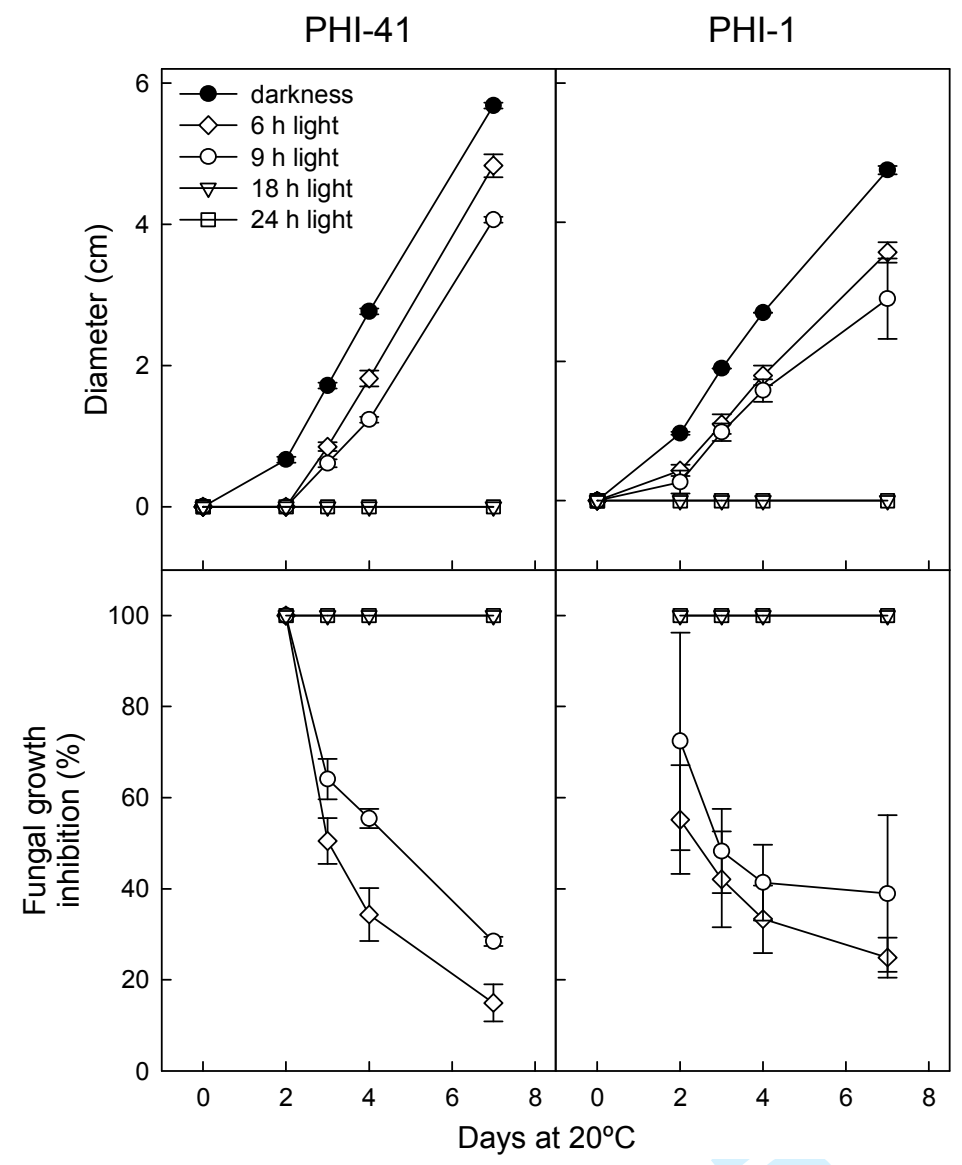




\section{Figure 6}

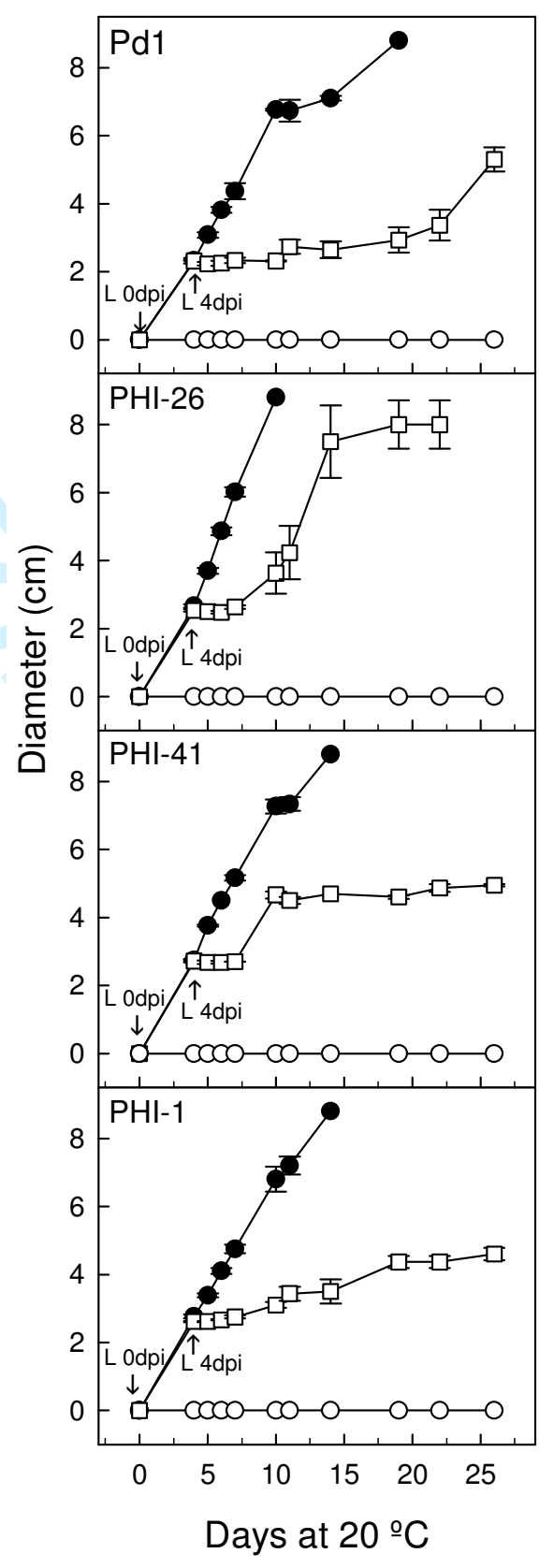


Fig. 7

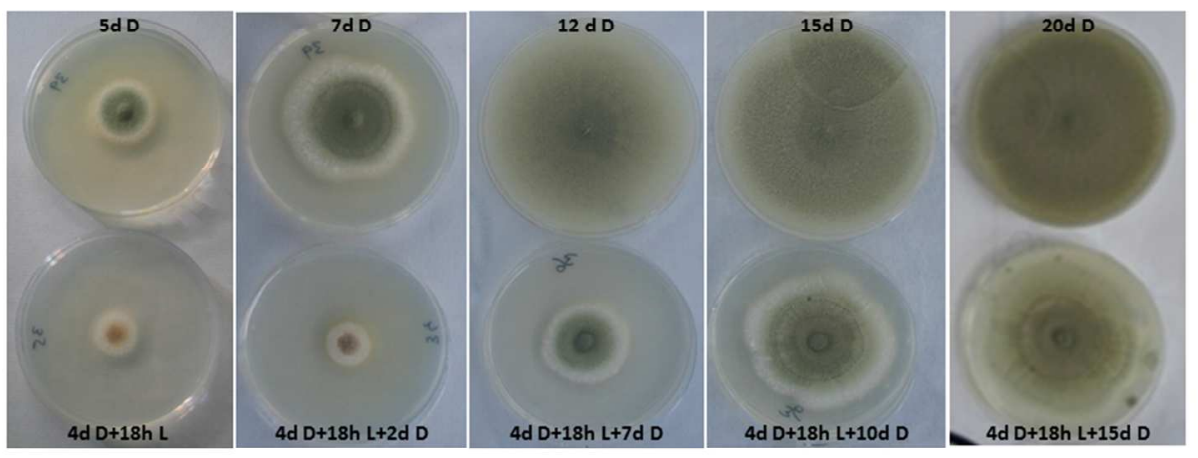

$254 \times 190 \mathrm{~mm}(96 \times 96 \mathrm{DPI})$ 